\title{
ANALYSIS OF FRP-REINFORCED CONCRETE BEAM WITH ARAMID SPIRALS AS COMPRESSION CONFINEMENT
}

\author{
H. Y. Leung ${ }^{1}$ and C. J. Burgoyne ${ }^{2}$ \\ ${ }^{1}$ Department of Building and Construction, City University of Hong Kong, \\ Tat Chee Avenue, Kowloon, Hong Kong \\ ${ }^{2}$ Department of Engineering, University of Cambridge, Trumpington Street, \\ Cambridge, United Kingdom
}

\begin{abstract}
This paper presents a method of analysis for the response of concrete beams reinforced with fibrereinforced-plastic (FRP) tension rods and aramid spirals. Three different kinds of longitudinal reinforcement are adopted; CFRP, AFRP and GFRP. Both under-reinforced and over-reinforced sections are described. It is found that the ratio of neutral axis depth and effective depth can be used as an indicator for FRP-reinforced beam with aramid spirals. Based on the computer solutions, a simple design chart is proposed.
\end{abstract}

\section{KEYWORDS}

Concrete, compression, aramid, spirals, confinement.

\section{INTRODUCTION}

FRPs are largely non-corrodable, light in weight and high in strength, so they are attractive as replacements for steel in reinforced concrete. Depending on the quantities of compression confinement and longitudinal reinforcement, failure may occur either in the compression region or the tensile bars. However, because FRPs exhibit linear elastic behaviour, it is desirable that the failure of flexural concrete members be controlled by concrete crushing. Such behaviour can be enhanced by the use of compression confinement, which significantly enhances the ultimate strain in compression.

\section{BACKGROUND}

The concept of a balanced section is used implicitly in designing steel-reinforced concrete structures (Kong and Evans 1992); the steel reinforcement reaches the yield strain simultaneously with the crushing strain being reached in the concrete. It is generally accepted that sections with steel reinforcement should be under-reinforced, so the steel yields before the concrete fails. The section is not the strongest that could be achieved by adding more reinforcement, but it has reasonable ductility. 
The moment capacity is affected very little by the strain capacity of the concrete, so there has been little interest in enhancing that capacity.

However, when FRPs are considered for use as the tension reinforcement, failure of that reinforcement would be by snapping, which is definitely to be avoided. Over-reinforced sections are thus desirable, and if their strain capacity can be enhanced that would lead to much higher deformability. This increase in strain capacity can be achieved by the use of spirals of FRP placed in the compression zone of the concrete, so that the lateral expansion of the concrete, caused by the axial stress, is resisted by the spirals, leading to triaxial stresses in the concrete. A typical layout can be found in Figure 1.

In this paper, the analytical prediction of the behaviour of a novel concrete beam reinforced with FRP spirals and tension rods is presented. Comparison will be made with three different types of FRP; carbon (C), aramid (A), and glass (G) FRPs. A concrete beam with a rectangular cross-section is investigated but the analysis can easily be extended to more complex sections.

\section{ANALYTICAL MODEL}

\section{Basic Assumptions}

The following assumptions are made. The ultimate strain of confined concrete, which is governed by snapping of the spiral, is accepted as the failure strain, rather than the strain at which the concrete reaches its maximum stress. A linear strain distribution is assumed across the full depth of the cross section. The tensile strength of concrete is ignored.

The distribution of the confined concrete compressive stress is found by considering the lateral strain induced in the concrete by the axial force, which in turn causes stresses in the spirals that enhance the strain capacity of the concrete. The full method, described in detail elsewhere (Leung 2000), is based on the hydrostatic and deviatoric stress-strain model by Kotsovos and Pavlovic (1995). Three different stress-strain curves result, one for the cover concrete, one for the concrete contained within a single spiral, and one for the concrete in the overlapping region, as shown in Figure 2. The stress in the FRP tension reinforcement is derived from its appropriate stress-strain profile and perfect bond is assumed.

Final failure occurs when the spiral snaps. This may not coincide with the maximum moment capacity of a section because the cover concrete will lose effectiveness before the confined concrete is carrying its maximum stress.

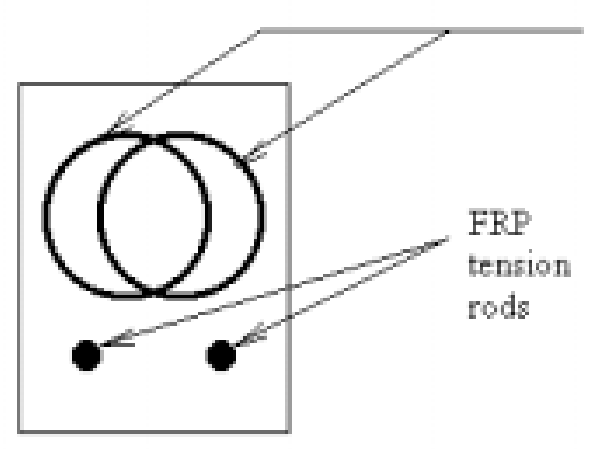

Figure 1: Novel beam design with rectangular cross section

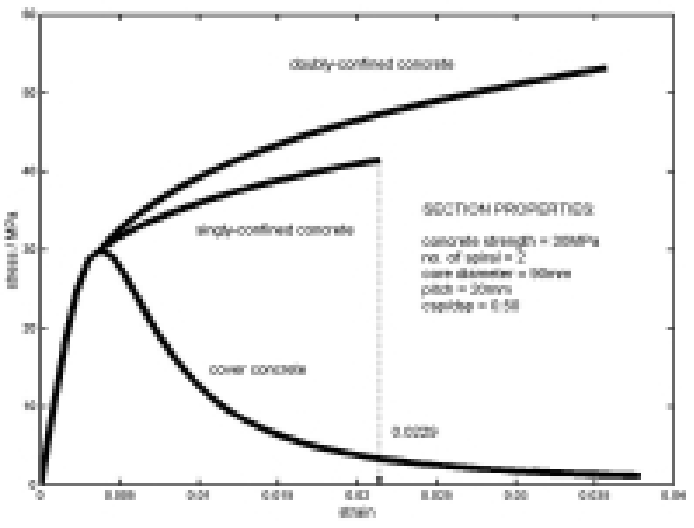

Figure 2: Stress strain curves for different concrete portions (Leung 2000) 
In order to find the area of FRP needed to achieve a balanced section, the neutral axis depth for the balanced case, $x_{b}$, can be calculated from simple trigonometry. If $d$ and $c^{\prime}$ represent the effective depth and spiral cover respectively (see Figure 3), it follows that:

$$
\frac{\varepsilon_{t}^{u}}{d-x_{b}}=\frac{\varepsilon_{c c}^{u}}{x_{b}-c^{\prime}}
$$

Rearranging,

$$
x_{b}=\frac{d+c^{\prime}\left(\frac{\varepsilon_{t}^{u}}{\varepsilon_{c c}^{u}}\right)}{1+\left(\frac{\varepsilon_{t}^{u}}{\varepsilon_{c c}^{u}}\right)}
$$

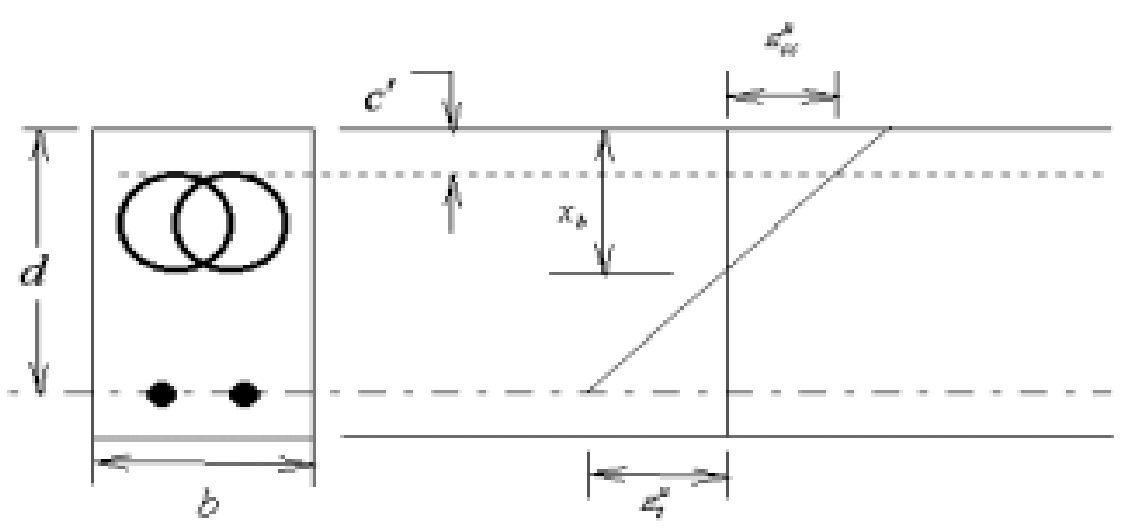

Figure 3: Balanced condition of novel beam design

$x_{b}$ is a function of the section geometry and the ultimate strains of both confined concrete $\left(\varepsilon_{c c}^{u}\right)$ and tensile reinforcement $\left(\varepsilon_{t}^{u}\right)$. To facilitate comparison, a section $300 \mathrm{~mm}$ deep with a width of $145 \mathrm{~mm}$ is considered, with a concrete cube strength of $30 \mathrm{MPa}$. The spiral is assumed to be made from aramid fibres with a fibre area of $1.75 \mathrm{~mm}^{2}$, ultimate strain of $1.53 \%$ and strength of $1.38 \mathrm{GPa}$. These values are lower than the bare fibre strength but allow for relatively large bundle effects. The spiral is assumed to have a pitch of $20 \mathrm{~mm}$ and an internal diameter of $90 \mathrm{~mm}$, with a centre-centre distance for the overlapping spirals of $45 \mathrm{~mm}$. For this geometry, the ultimate strain of confined concrete is 0.0229 (Leung 2000).

TABLE 1

COMPARISON OF DIFFERENT TYPES OF FRP TENSION BARS

\begin{tabular}{|c|c|c|c|}
\hline Material & $\begin{array}{c}\text { CFRP } \\
\text { (resin }+ \text { fibre) }\end{array}$ & $\begin{array}{c}\text { AFRP } \\
\text { (resin }+ \text { fibre) }\end{array}$ & $\begin{array}{c}\text { GFRP } \\
\text { (resin + fibre) }\end{array}$ \\
\hline Symbol & $\mathrm{O}$ & + & $*$ \\
\hline Ultimate strain $\varepsilon_{t}^{u}$ & 0.017 & 0.021 & 0.023 \\
\hline Ultimate strength $\sigma_{t}^{u}$ & $2862 \mathrm{MPa}$ & $1400 \mathrm{MPa}$ & $1103 \mathrm{MPa}$ \\
\hline Elastic modulus & $168 \mathrm{GPa}$ & $67 \mathrm{GPa}$ & $48 \mathrm{GPa}$ \\
\hline$x_{b} / d$ & 0.58 & 0.53 & 0.51 \\
\hline
\end{tabular}


The mechanical properties of three typical FRP composites are given in Table 1 (Nanni 1993 and Saadatmenesh et al 1994) and the corresponding values of $x_{b} / d$ are calculated. The stress-strain curves of CFRP, AFRP and GFRP are all linear. As expected, the material with the highest strain capacity (GFRP) gives the smallest value of $x_{b}$.

\section{Over-reinforced Analysis of Rectangular Section}

To carry out the analysis at the ultimate state, the concrete section is first divided into several thin slices. When two spirals are placed inside the compression region, a maximum of three different concrete types can be formed, (1) the unconfined cover concrete, (2) singly-confined concrete and (3) doubly-confined concrete. Depending upon the geometrical configurations, each concrete slice may contain up to three different characters of concrete, each with its own stress-strain relationship.

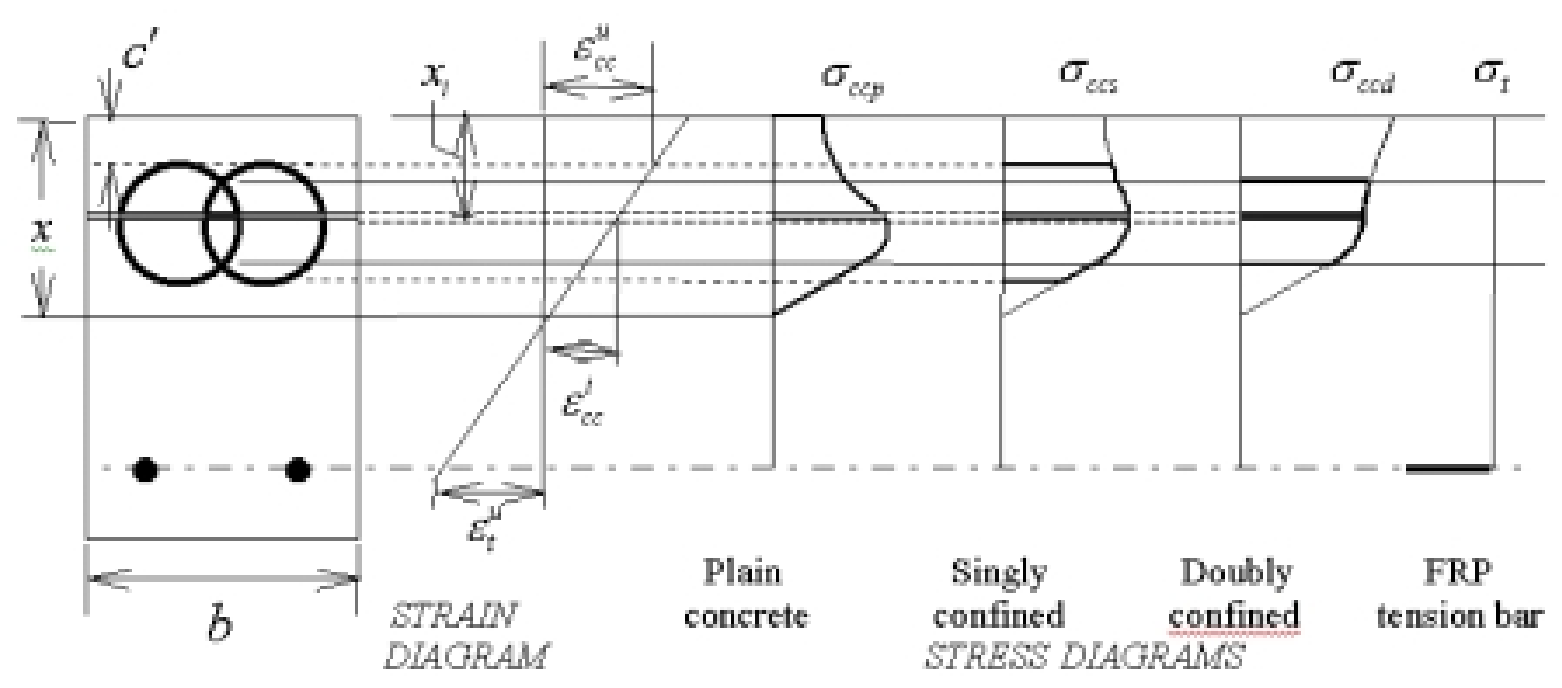

Figure 4: Strain and stress diagrams

If the area of the tension reinforcement is known, it is necessary to iterate to satisfy longitudinal force equilibrium. The strain at the top of the singly-confined concrete is fixed at $\varepsilon_{c c}^{u}$. The position of the neutral axis is then allowed to vary. However, in this analysis the position of the neutral axis is fixed and the amount of reinforcement required is obtained by calculation. This allows the production of charts showing the behaviour of beams in different circumstances. The strain value of each slice $\left(\varepsilon_{c c}^{i}\right)$ can be found from Figure 4

$$
\varepsilon_{c c}^{i}=\frac{x-x_{i}}{x-c^{\prime}} \varepsilon_{c c}^{u}
$$

The corresponding concrete stress $\left(\sigma_{c c}^{i}\right)$ can be found. If the slice cuts across three different regions of concrete, three different values of $\sigma_{c c}^{i}$ are found. The values of strain in the tension reinforcing bar $\left(\varepsilon_{t}\right)$ can now be described in terms of the concrete strain and neutral axis depth, and the stress found from its stress-strain relationship.

$$
\varepsilon_{t}=\left(\frac{d-x_{b}}{x_{b}-c^{\prime}}\right) \varepsilon_{c c}^{u}=\varepsilon_{t}^{u}
$$

and

$$
\sigma_{t}=E_{t} \varepsilon_{t}=\sigma_{t}^{u}
$$


The resultant compressive force of the concrete section can be calculated by summing the corresponding products of the elemental area and stress values.

$$
\begin{aligned}
& F_{c c p}=\sum_{i} A_{c c p}^{i} \sigma_{c c p}^{i} \\
& F_{c c s}=\sum_{i} A_{c c s}^{i} \sigma_{c c s}^{i} \\
& F_{c c d}=\sum_{i} A_{c c d}^{i} \sigma_{c c d}^{i}
\end{aligned}
$$

where the subscripts $c c p, c c s$ and $c c d$ represent the unconfined, singly-confined and doubly-confined concrete portions, respectively. The total compressive force is then:

$$
F_{c c}=F_{c c p}+F_{c c s}+F_{c c d}
$$

The tensile force in the FRP tensile reinforcement $\left(F_{t}\right)$ is:

$$
F_{t}=A_{t} \sigma_{t}
$$

where $A_{t}$ indicates the total cross sectional area of the FRP bars.

Applying the principle of force equilibrium, and neglecting the tensile capacity of concrete, the compressive resultant force is identical to the tensile force generated in the FRP rods, i.e.

$$
F_{t}=F_{c c}
$$

Each resultant compressive force produces a moment about the tension bar. To find the value of the bending moment, for each slice, the bending moment is calculated by multiplying the force and the lever $\operatorname{arm}(z$ with appropriate subscripts).

$$
M=\sum_{i} F_{c c p}^{i} z_{c c p}^{i}+\sum_{i} F_{c c s}^{i} z_{c c s}^{i}+\sum_{i} F_{c c d}^{i} z_{c c d}^{i}
$$

The ultimate design moment is thus related to the resultant tensile force by:

$$
M=F_{t} z_{t}
$$

where $z_{t}$ represents the lever arm between the total resultant compressive force and the tensile force:

$$
z_{t}=\frac{\sum_{i} F_{c c p}^{i} z_{c c p}^{i}+\sum_{i} F_{c c s}^{i} z_{c c s}^{i}+\sum_{i} F_{c c d}^{i} z_{c c d}^{i}}{\sum_{i} F_{c c p}^{i}+\sum_{i} F_{c c s}^{i}+\sum_{i} F_{c c d}^{i}}
$$

The required reinforcement area and the reinforcement ratio can then be found by:

$$
A_{t}=\frac{M}{\sigma_{t}^{u} z_{t}}
$$

and 


$$
\frac{A_{t}}{b d}=\frac{M}{\sigma_{t}^{u} z_{t} b d} .
$$

The curvature can also be obtained by dividing the maximum concrete strain by the neutral axis depth.

\section{Under-reinforced Analysis}

If $x<x_{b}$, the section will be under-reinforced. In this case, the FRP reaches its limit strain $\varepsilon_{t}^{u}$ before the concrete reaches $\varepsilon_{c c}^{u}$. A similar analysis can be carried out for this case, fixing the FRP strain at $\varepsilon_{t}^{u}$

$$
\varepsilon_{c c}^{i}=\frac{x-x_{i}}{d-x} \varepsilon_{t}^{u}
$$

The analysis then proceeds as before.

\section{SPECIAL FEATURES OF THE BEAM DESIGN}

Results of analyses applied to the section are shown in Figures 5 to 8 . Non-dimensional moment (defined by $M /\left(\sigma_{c}^{u} b d^{2}\right)$ ), FRP area (defined by $A_{t} /(b d)$ ) and curvature (defined by $\kappa d$ ) are plotted against $x / d$ for all three materials (Table 1 ). The balanced section is indicated by $\mathrm{O}$ for CFRP, + for AFRP and $*$ for GFRP. Over-reinforced sections lie to the right of these points and under-reinforced sections to the left in all cases.

From the Figure 5, it is clear that when the beam is designed to be over-reinforced, the moment capacity is independent of the tension bar material. In contrast, when the value of $x / d$ is lower than its balanced value, the moment capacity depends on the material. When the section is underreinforced, the FRP bar fails first and its ultimate strain capacity controls the ultimate condition of the entire section. GFRP has the highest ultimate strain value while CFRP has the lowest strain capacity amongst the three FRPs.

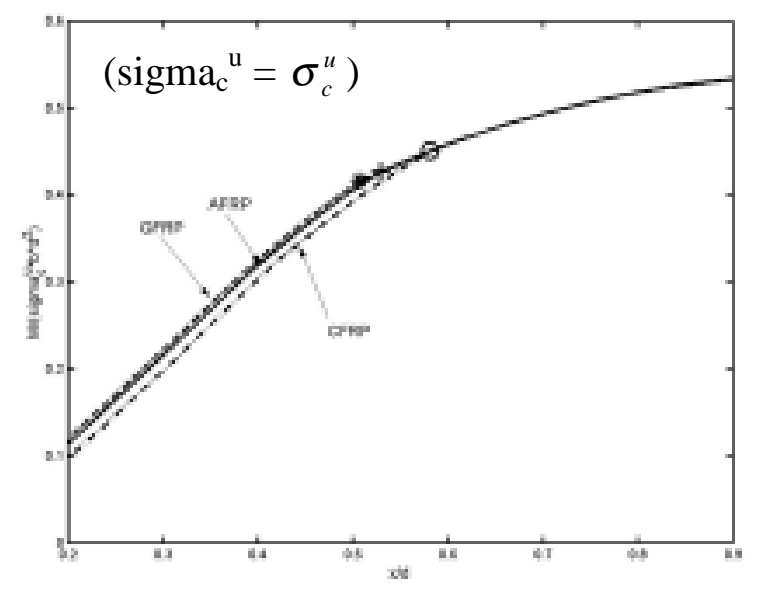

Figure 5: Moment capacity

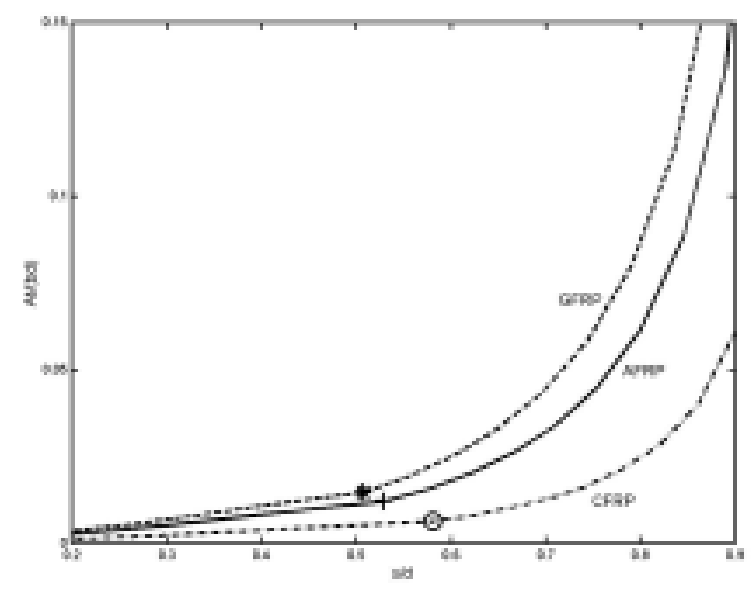

Figure 6: FRP area required

When the area of FRP required is considered, as in Figure 6, the strength of the tendon is important so more GFRP is required than CFRP. The amount of GFRP required is, however, impractically high. If 
$A_{t}$ is non-dimensionalised by taking into account the Young's modulus $E_{t}\left(A_{t} E_{t} /\left(100 b d \sigma_{c}^{u}\right)\right)$, the same behaviour in the over-reinforced region is noted for all materials (Figure 7).

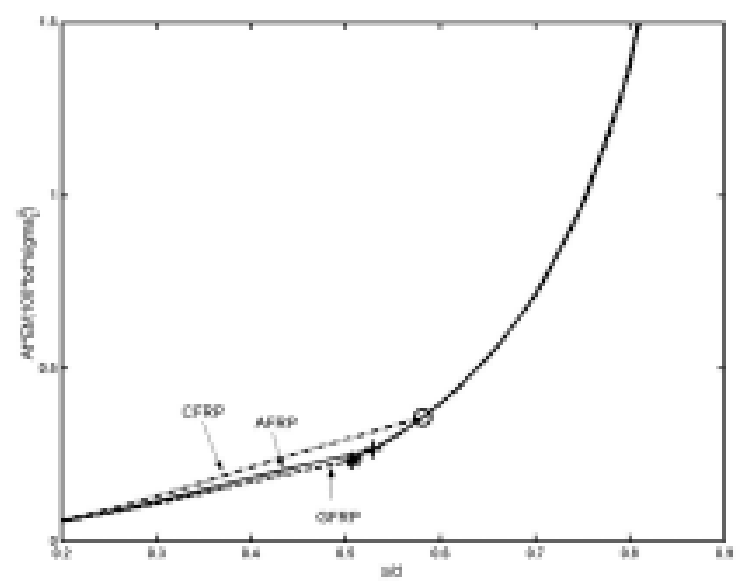

Figure 7: FRP area required (modified)

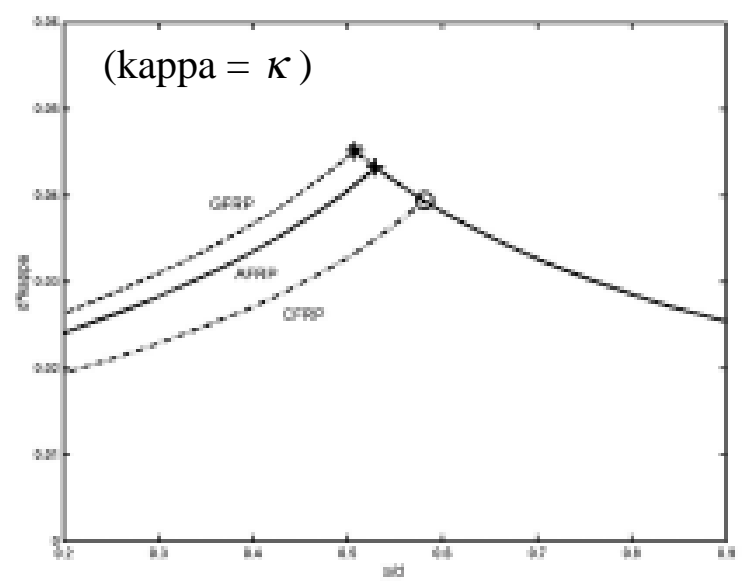

Figure 8: Curvature

In Figure 8, the curvature is shown. As expected GFRP shows higher curvature than CFRP. As with the moment capacity, and for the same reason, the materials show the same behaviour when overreinforced. The highest curvature capacity occurs at the balanced condition.

\section{MOMENT-CURVATURE BEHAVIOUR}

A similar analysis has been carried out with fixed $A_{t}$. The strain in the concrete is varied and the neutral axis position iterated until longitudinal equilibrium is satisfied. This allows complete momentcurvature relation to be plotted, as shown in Figure 9.

Figure 9(a) shows the relationship of moment against curvature for the balanced condition. All three FRPs show a similar increasing profile. However, CFRP indicates the lowest curvature capacity while GFRP gives the highest; the ultimate tensile strain again governs the variation. On the other hand, CFRP produces the highest moment while GFRP is the lowest; this is governed by the elastic modulus. In Figure 9(b), the amount of tension reinforcement is taken as twice that used in the balanced condition, so that an over-reinforced condition applies. A slight increase in moment is observed. The same overall trend applies, but the ultimate curvatures are reduced. This reduction in curvature capacity is also reflected in Figure 9(c) in which the under-reinforced condition is considered with $A_{t}=A_{t b} / 2$. In addition, a great decrease of moment capacity is observed.

In order to give a clear picture for design purposes, the variation of $\frac{M}{\sigma_{c}^{u} b d^{2}}$ against $\frac{A_{t} E_{t}}{100 b d \sigma_{c}^{u}}$ for the three FRPs is produced as in Figure 10 for the current concrete and spiral details. As seen in the figure, when the design moment is known, the required amount of FRP tension reinforcement can be deduced from the graph. Apart from the balanced condition, the present design chart is applicable to both under and over-reinforced situations. It is significant that the curves are very flat in the overreinforced region, which implies that there is a fairly narrow band of section sizes which are desirable for any applied movement. The chart is specific to the size and shape of beam that has been considered here, but very similar charts could be produced easily for different spirals or different shapes of cross-section. 

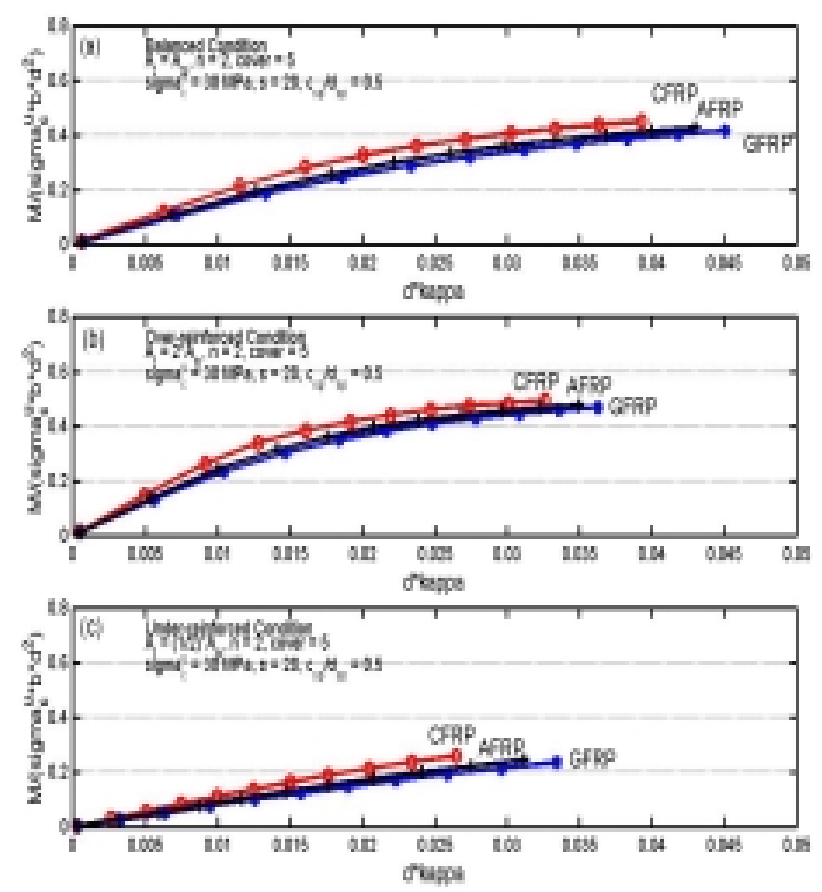

Figure 9: Variation of moment term against curvature term for (a) balanced, (b) over- (c) under-reinforced conditions

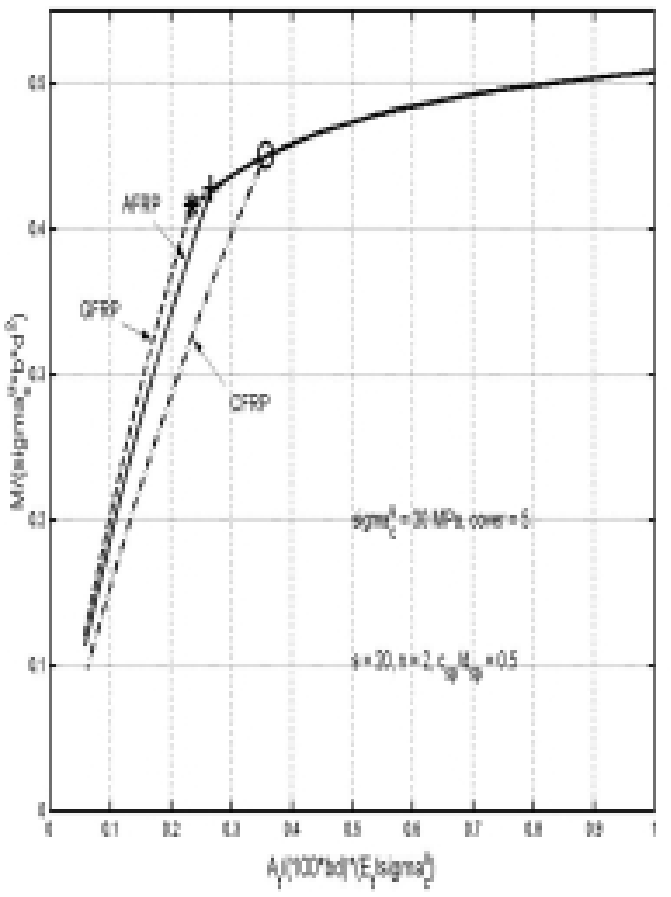

Figure 10: Design chart

\section{CONCLUSIONS}

A novel beam design with FRP spirals inside the compression flange is found to be feasible. Typical analysis and design procedures of such novel beam have been presented. Three different types of FRPs have been used to illustrate the subtle variations.

It has been concluded that a non-dimensional ratio of $x / d$ can be used as an indicator for different characteristics of the novel beam behaviour. The under-reinforced section behaves differently when compared to the over-reinforced design. Nevertheless, a remarkable enhancement in moment and strain capacity is anticipated for over-reinforced novel beam design.

\section{REFERENCES}

Leung, H.Y. (2000) Aramid fibre spirals to confine concrete in compression, $\mathrm{PhD}$ thesis, University of Cambridge, 200pp.

Kong, F.K. and Evans, R.H. (1992) Reinforced and Prestressed Concrete, Chapman \& Hall, 508pp.

Kotsovos, M.D. and Pavlovic, M.N. (1995) Structural concrete, Thomas Telford, 549pp.

Nanni, A. (1993) "Flexural Behavior and Design of RC Members Using FRP Reinforcement", $J$. Struct. Engng., ASCE, 119, No. 11, Nov., pp.3344-3359.

Saadatmanesh, H., Ehsani, M.R. and Li, M.W., (1994) "Strength and Ductility of Concrete Columns Externally Reinforced with Fiber Composite Straps”, ACI Struct. J., 91, Jul.-Aug., pp.434-447. 